\title{
Editorial
}

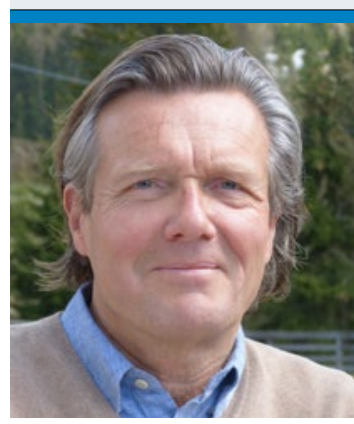

Prof. Dr. med.

Gerhard Grevers

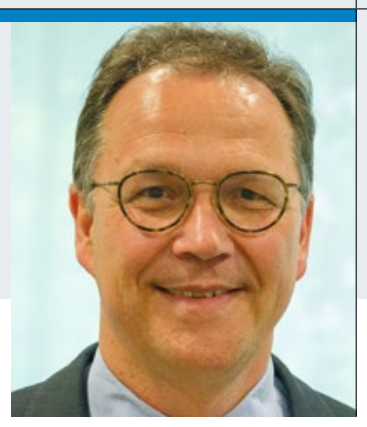

"Zwei Beiträge in diesem Heft (S. 29 und S. 46) möchten

wir Ihnen besonders ans Herz legen, veranschaulichen

sie doch an Hand aktueller Beispiele die fragwürdige

Geldverteilungspolitik der GKV."

\section{Fragwürdige Neuerungen}

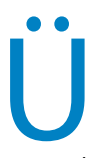

ber den Wildwuchs im Medizin-App-Geschäft hatten wir bereits im Frühsommer vergangenen Jahres berichtet (HNO-Nachrichten 3/2016). Ein schönes Beispiel für eine fragwürdige Empfehlung ist in diesem Zusammenhang die Tinnitus-App „Tinnitracks“, die von der Vertreiberfirma Sonormed schon seit einiger Zeit mit Hochglanzprospekten und HNO-Fortbildungsveranstaltungen in mehreren Bundesländern recht ambitioniert beworben wird. Schützenhilfe kommt auch vom Deutschen Ärzteblatt (Heft 42/2016) und den HNOMitteilungen (Heft 6/2016); die Lobbyarbeit scheint also schon mal zu funktionieren. Die Techniker Krankenkasse, die seit Ende 2015 die Kosten für die App übernimmt, geht allerdings bei der Unterstützung dieser Anwendung noch einen Schritt weiter, wenn sie in ihrer Hauspostille unter der Überschrift "Pop gegen das Piepen im Ohr" (TK aktuell 2/2016, S. 16) „Tinnitracks" als „,innovative Behandlungsmethode" beschreibt und sich dabei u. a. auch auf „neue wissenschaftlicher Erkenntnisse" beruft. Starker Tobak, der belegbar sein sollte. Der Geschäftsführer der Firma Sonormed, über die „Tinnitracks“vertrieben wird, ein gewisser Herr Land, tingelt derweil werbewirksam in Sachen eHealth-Industrie durch die Republik, mal auf dem Wirtschaftsgipfel der SZ mit Gesundheitsminister Gröhe, mal auf einer Veranstaltung namens "Chasing Giants - How Health Start-ups and Industry leaders work together" mit dem ehemaligen Gesundheitsminister und heutigen Allianz-Lobbyisten Bahr. Giganten unter sich halt, kleiner geht's in der Branche nicht. Auch im oben zitierten Ärzteblattartikel bläst Land ordentlich die Backen auf: „Ganz neue Technologien und Ideen dringen in eine Branche ein, die nicht unbedingt auf so viel Dynamik vorbereitet ist“". Die Ärzte, Schlafmützen eben. Nun ja, in der Start-up-Community macht man eben gerne mal auf „dicke Hose“; wo das hinführt, haben wir am „Neuen Markt“ gesehen, aber das ist ja auch schon wieder eine Weile her. Gerhard Goebel und Gerhard Hesse, zwei seit Jahrzehnten ausgewiesene Tinnitusexperten, haben sich „Tinnitracks" einmal hinsichtlich Innovativität und wissenschaftlich belegbarer Se- riosität etwas genauer angeschaut und kommen in ihrer Bewertung zu ganz anderen Schlüssen als die „Experten" der Techniker Krankenkasse und der vollmundige Herr Land (s. S. 29 in diesem Heft).

Werden Versichertengelder allzu leichtfertig verplempert, fehlen sie an anderen Stelle, wo man sie durchaus sinnvoller einsetzen könnte.

Damit sind wir bei unserem zweiten Beispiel, einer erneuten Änderung in der Verschreibungsfähigkeit von Antiallergika. Antihistaminika sind ja seit 2006 (fast) nicht mehr zulasten der GKV verordnungsfähig. Seit dem 15.10.2016 sind nun wichtige nasale Glukokortikoide ebenfalls nicht mehr über das "rote“ Rezept verordnungsfähig (Beitrag S. 46). Auch wenn dies zunächst nur für die Behandlung der saisonalen allergischen Rhinitis (AR) nach Erstdiagnose durch den Arzt in einer maximalen Tagesdosis von 400/200 Mikrogramm und nur für Erwachsene gilt, ist abzusehen, dass diese Änderung die Versorgung von Patienten mit nasalen Allergien weiter erheblich beeinträchtigen wird. Wer will denn die Apotheker dahingehend kontrollieren, ob die genannten Einschränkungen tatsächlich vorliegen? Weder kann der Apotheker prüfen, ob eine ärztliche Erstdiagnose tatsächlich erfolgte, noch wird die Art der Diagnose (nur erlaubt bei saisonaler AR) kontrollierbar sein. Die Folgen sind absehbar. Wenn es kaum noch Medikamente gibt, die wir den Patienten auf GKV-Rezept verordnen können, wird die primäre Behandlung immer mehr auf die Apotheker übergehen. Studien belegen eindeutig, dass dies zu einer schlechteren Versorgung führt. Des Weiteren werden die Patienten zukünftig noch viel später (evtl. auch gar nicht) über die allergenspezifische Immuntherapie als einzige kausale Behandlungsoption der AR informiert. Durch eine Chronifizierung der Erkrankung, Etagenwechsel und Ausweitung des Sensibilisierungsspektrums sind zudem erhebliche finanzielle Mehrbelastungen für die Sozialsysteme programmiert. Eine frühzeitige effektive Therapie wäre demgegenüber á la longue nicht nur kostengünstiger, sondern würde den Patienten unnötige zusätzliche Beschwerden ersparen. Logisch eigentlich. 